\title{
Virtual Monoenergetic Images from Spectral Detector CT Enable Radiation Dose Reduction in Unenhanced Cranial CT
}

\author{
(D) R.P. Reimer, (DD. Flatten, (D)T. Lichtenstein, (D) D. Zopfs, (D) V. Neuhaus, (D) C. Kabbasch, (DD. Maintz, (D). Borggrefe, and
}

(iD) Große Hokamp

\begin{abstract}
BACKGROUND AND PURPOSE: Our aim was to evaluate whether improved gray-white matter differentiation in cranial CT by means of $65-\mathrm{keV}$ virtual monoenergetic images enables a radiation dose reduction compared to conventional images.

MATERIALS AND METHODS: One hundred forty consecutive patients undergoing 171 spectral detector CTs of the head between February and November 2017 (56 \pm 19 years of age; male/female ratio, 56\%/44\%) were retrospectively included. The tube currenttime product was reduced during the study period, resulting in 61, 55, and 55 patients being examined with 320, 290, and 260 mAs, respectively. All other scanning parameters were kept identical. The volume CT dose index was recorded. ROls were placed in gray and white matter on conventional images and copied to identical positions in 65-keV virtual monoenergetic images. The contrastto-noise ratio was calculated. Two radiologists blinded to the reconstruction technique evaluated image quality on a 5-point Likert-scale. Statistical assessment was performed using ANOVA and Wilcoxon test adjusted for multiple comparisons.
\end{abstract}

RESULTS: The mean volume CT dose index was 55, 49.8, and $44.7 \mathrm{mGy}$ using 320, 290, and $260 \mathrm{mAs}$, respectively. Irrespective of the volume CT dose index, noise was significantly lower in $65-\mathrm{keV}$ virtual monoenergetic images compared with conventional images (65- keV virtual monoenergetic images/conventional images: extraocular muscle with $49.8 \mathrm{mGy}, 3.7 \pm 1.3 / 5.6 \pm 1.6 \mathrm{HU}$, $P<.001)$. Noise slightly increased with a reduced radiation dose (eg, extraocular muscle in conventional images: $5.3 \pm 1.4 / 5.6 \pm 1.6 /$ $6.1 \pm 2.1 \mathrm{HU})$. Overall, the contrast-to-noise ratio in $65-\mathrm{keV}$ virtual monoenergetic images was superior to that in conventional images irrespective of the volume CT dose index $(P<.001)$. Particularly, $65-\mathrm{keV}$ virtual monoenergetic images with $44.7 \mathrm{mGy}$ showed significantly lower noise and a higher contrast-to-noise ratio than conventional images with $55 \mathrm{mGy}(P<.001)$. Subjective analysis confirmed better image quality in $65-\mathrm{keV}$ virtual monoenergetic images, even using $44.7 \mathrm{mGy}$.

CONCLUSIONS: The 65-keV virtual monoenergetic images from spectral detector CT allow radiation dose reduction in cranial CT. While this proof of concept included a radiation dose reduction of $19 \%$, our data suggest that even greater reduction appears achievable.

ABBREVIATIONS: $\mathrm{Cl}=$ conventional images; $\mathrm{CNR}=$ contrast-to-noise ratio; $\mathrm{CTDI}_{\mathrm{vol}}=$ volume $\mathrm{CT}$ dose index; $\mathrm{VMI}=$ virtual monoenergetic images

U nenhanced cranial CT is the standard examination for patients with acute neurologic deficits to allow fast diagnosis of emergencies, for instance, intracranial hemorrhage or ischemia. ${ }^{1-4}$ There are approximately 70 million cranial CT scans annually in the United States alone; out of these, several scans are performed in the same patient, so that they undergo

Received May 17, 2019; accepted after revision August 5.

From the Department of Diagnostic and Interventional Radiology, University of Cologne, Faculty of Medicine and University Hospital Cologne, Cologne, Germany.

R.P. Reimer and D. Flatten contributed equally to this work.

Please address correspondence to Nils Große Hokamp, MD, Department of Diagnostic and Interventional Radiology, University Hospital Cologne, Cologne, Germany, Kerpener Str 62, 50937 Köln, Germany; e-mail:

nils.grosse-hokamp@uk-koeln.de

http://dx.doi.org/10.3174/ajnr.A6220 repetitive scanning. ${ }^{5}$ Despite rapid advances in the field of CT imaging such as dose modulation or iterative image reconstruction, few of these have been applied to cranial CT for 2 main reasons: First, there are only subtle differences in attenuation between gray and white matter. Yet, this is one of the most important aspects to evaluate, in particular in light of suspected ischemia. Second, the surrounding skull causes beam-hardening and therefore an increase in image noise due to beam-hardening. ${ }^{6-9}$ Hence, possibilities for dose reduction are limited, despite radiosensitive tissues such as the eye lenses being exposed. $^{10-13}$

A recent development in the field of CT is dual-energy CT, which has been evolving for the past decade. Dual-energy CT is known to improve soft-tissue contrast by means of virtual monoenergetic images (VMI). ${ }^{14-16}$ These VMI further reduce artifacts 


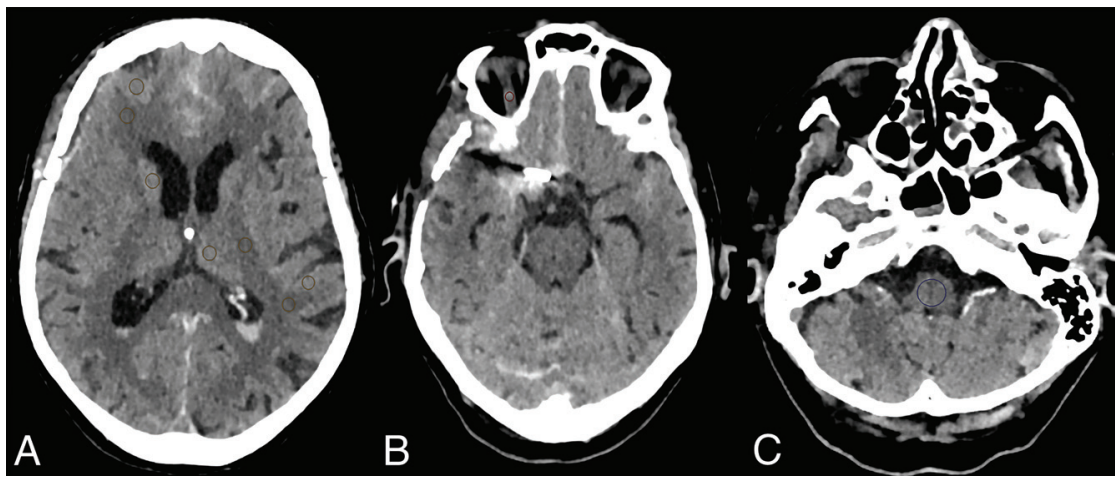

FIG 1. ROI placement in the cortical gray and juxtacortical white matter, in the thalamic parenchyma and posterior limb of the internal capsule, in the caudate nucleus (orange ROIs), in an extraocular muscle (red $R O I)$, and in the medulla oblongata (blue $R O I$ ) on an axial plane showing the basal ganglia $(A)$, the orbital cavity $(B)$, and the posterior fossa $(C)$.

occurring due to beam-hardening. In light of neuroimaging, dual-energy CT demonstrated improved image quality and lesion characterization, while it also allowed material separation for iodine. $^{15,17-22}$

Dual-energy CT systems register low- and high-energy data attenuation profiles. By linear blending of these datasets, VMI can be reconstructed. VMI represent virtually approximated images, which would result from acquisition with a true monoenergetic $\mathrm{x}$-ray beam. They are typically available in a range from 40 to $200 \mathrm{keV}$, depending on the dual-energy CT system used. ${ }^{23,24}$

Different emission-based dual-energy CT systems have been available for several years using emission spectra with lower and higher mean energy. ${ }^{25,26}$ More recently, a detector-based approach was introduced, referred to as spectral detector CT. Here, low- and high-energy photons are registered separately using a dual-layer detector. ${ }^{24,26}$ The upper layer is yttrium-based and registers lower energy photons, while the lower layer is gadolinium oxysulfide-based, registering higher energy photons. ${ }^{8,24,26}$

In a recent study, VMI from spectral detector CT showed superior image quality in examinations of the head compared with conventional images (CI). Corticomedullary differentiation was found to be best in $65-\mathrm{keV}$ VMI $\left(\mathrm{VMI}_{65 \mathrm{keV}}\right)$, while in lower kiloelectron volt images, beam-hardening artifacts close to the calvaria distorted image quality. ${ }^{6}$ Their data suggest a VMIenabled radiation dose reduction.

Therefore, the aim of our study was to compare $\mathrm{VMI}_{65 \mathrm{keV}}$ with CI from unenhanced spectral detector CT datasets of the head acquired with different acquisition protocols to evaluate whether improved image quality in $\mathrm{VMI}_{65 \mathrm{kev}}$ allows a reduction of radiation dose.

\section{MATERIALS AND METHODS}

To meet national requirements for radiation dose, we modified protocols for cranial CT examinations, including a reduction in the radiation dose. The institutional review board later approved the scientific evaluation of these data and waived informed consent due to the retrospective study design. A structured search in the radiology information system was performed with the following inclusion criteria: 1) older than 18 years of age, 2) an unenhanced spectral detector CT of the head between the February 1,
2017, and November 30, 2017, and 3) a standardized imaging protocol as described below. Exclusion criteria were the following: 1) extensive intracranial hemorrhage or edema, 2) craniectomy or hemicraniectomy, and 3) artifacts due to patient movement or implants. Eventually, 140 patients with 171 CT scans were included in this study.

\section{Acquisition Parameters}

All CT scans were performed for clinical indications on the same spectral detector CT scanner (IQon Spectral CT; Philips Healthcare, Best, the Netherlands). Sixty-one of the identified CT scans were obtained with a tube current-time product of $320 \mathrm{mAs}$, 55 with $290 \mathrm{mAs}$, and 55 with $260 \mathrm{mAs}$. All other scan parameters were kept identical: tube voltage $=120 \mathrm{kV}$ (peak), pitch $=0.36$, rotation time $=0.33$ seconds, and collimation $=64 \times 0.625$. CI were reconstructed using a hybrid iterative reconstruction algorithm (iDose4, Filter UB; Philips Healthcare). $\mathrm{VMI}_{65 \mathrm{keV}}$ were reconstructed using a dedicated spectral image-reconstruction algorithm (Spectral, Filter UB; Philips Healthcare). Denoising for both was set to a medium level (level 3 of 7). All images were reconstructed with a section thickness of $1 \mathrm{~mm}$ and a section increment of $1 \mathrm{~mm}$.

Dose-length product and volume CT dose index $\left(\mathrm{CTDI}_{\mathrm{vol}}\right)$ were recorded from the radiation dose report. We further compared the anterior-posterior and lateral dimensions of the head between groups to exclude this as a confounder.

\section{Quantitative Analysis}

Quantitative analysis was performed using ROI-based measurements of attenuation and SD in the following areas on a representative axial plane: 1) cortical gray and 2) adjacent juxtacortical white matter of the frontal and parietal lobes, 3) thalamic parenchyma, 4) adjacent posterior limb of the internal capsule, 5) caudate nucleus, 6) extraocular muscle, and 7) medulla oblongata (Fig 1).

ROIs were placed on CI and copied to identical positions in $\mathrm{VMI}_{65 \mathrm{keV}}$. The size of the ROIs was kept constant at $25 \mathrm{~mm}^{2}$, except for the ROI in the medulla oblongata $\left(100 \mathrm{~mm}^{2}\right)$, and was only adjusted to avoid inclusion of unrepresentative tissue. One radiologist with 2 years of experience in cranial CT interpretation performed the quantitative analysis. In a randomly chosen subgroup of 30 cranial CT scans, a second reader repeated the ROI placement to assess interrater reliability.

Image noise was considered as an SD of extraocular muscle. The contrast-to-noise ratio (CNR) of the gray and white matter of the frontal and parietal lobes was calculated as the difference of the average Hounsfield unit, divided by the square root of the sum of the SD of the 2 adjacent ROIs. ${ }^{6,15}$

\section{Qualitative Analysis}

Qualitative analysis was performed independently by 2 fellowship-trained trained neuroradiologists. Readers were blinded to 
the reconstruction technique. Rating was performed on 5-point Likert scales with regard to assessment of gray-white matter differentiation in the following areas: 1) the basal ganglia, 2) the supratentorial cortex, 3) the infratentorial cortex, and 4) the subcalvarial space $(1=$ not diagnostic; $2=$ severely impaired assessment; 3 =moderate assessment; 4 =fair assessment; $5=$ good assessment, fully diagnostic). Furthermore, visually perceived image noise and beam-hardening artifacts in the subcalvarial space were evaluated $(1=$ excessive; $2=$ severe; $3=$ moderate; 4 = some; $5=$ no visually perceptible noise).

\section{Statistical Analysis}

All analyses were performed using JMP Software (Version 12; SAS Institute, Cary, North Carolina) unless specified below. To compare groups, we used ANOVA or Wilcoxon tests, adjusted for multiple comparisons if appropriate. A $P$ value $<$ .05 was considered significant. Results are shown as mean \pm $\mathrm{SD}$. Interrater reliability was determined by means of intraclass correlation estimates using $\mathrm{R}$ Studio (Version 1.1.456; http://rstudio.org/download/desktop) based on a single rater, consistency, 2-way mixed-effects model for the quantitative analysis and based on a mean of 2 raters, consistency, 2-way mixed-effects model for the qualitative analysis. ${ }^{27}$ Interrater agreement was evaluated as described earlier: excellent (intraclass correlation coefficient $>0.8$ ), good (intraclass correlation coefficient $>0.6$ ), moderate (intraclass correlation coefficient $>0.4$ ), and poor agreement (intraclass correlation coefficient $\leq 0.4)^{28,29}$

Table 1: Radiation dose

\begin{tabular}{lccc}
\hline Tube Current-Time Product (mAs) & $\mathbf{3 2 0}$ & $\mathbf{2 9 0}$ & $\mathbf{2 6 0}$ \\
\hline DLP $(\mathrm{mGy} \times \mathrm{cm})^{\mathrm{a}}$ & $1014.9 \pm 56.9$ & $937.7 \pm 40.2$ & $837.7 \pm 45.6$ \\
Radiation dose reduction & & $-7.6 \%$ & $-17.5 \%$ \\
$\mathrm{CTDI}_{\text {vol }}(\mathrm{mG}$ ) & 55 & 49.8 & 44.7 \\
Radiation dose reduction & & $-9.5 \%$ & $-18.7 \%$ \\
\hline
\end{tabular}

Note:-DLP indicates dose-length product.

${ }^{\text {a }}$ Results are means \pm SDs.

\section{RESULTS}

The mean age of patients was $55.8 \pm 18.6$ years; of these patients, $61(43.6 \%)$ were women and $79(56.4 \%)$ men.

\section{Radiation Dose}

CTDI $_{\text {vol was }} 55,49.8$, and $44.7 \mathrm{mGy}$ in examinations with 320 , 290 , and $260 \mathrm{mAs}$, respectively. The dose-length product was $1014.9 \pm 56.9,937.7 \pm 40.2$, and $837.7 \pm 45.6 \mathrm{mGy} \times \mathrm{cm}$ $(P<.001)$ (Table 1$)$. Regarding the $\mathrm{CTDI}_{\mathrm{vol}}$, the radiation dose was reduced by $9.5 \%$ and $18.7 \%$. No significant differences in head size between groups were found $(P \geq .05)$.

\section{Quantitative Analysis}

The intraclass correlation between the 2 independent readers was 0.984 with a $95 \%$ confidence interval of $0.982-0.985$, indicating an excellent interreader reliability.

\section{Attenuation}

For the same $\mathrm{CTDI}_{\mathrm{vol}}$, attenuation in gray matter was significantly higher in $\mathrm{VMI}_{65 \mathrm{keV}}$ compared with $\mathrm{CI}(P \leq .01$ (Table 2). On the other hand, attenuation in white matter was slightly higher in CI compared with $\mathrm{VMI}_{65 \mathrm{keV}}$ for 55- and 49.8-mGy protocols without reaching a significant difference $(P>.05)$, while for $44.7 \mathrm{mGy}$, it was slightly higher in $\mathrm{VMI}_{65 \mathrm{keV}}$ compared with $\mathrm{CI}(P>.05)$

\section{Noise}

Image noise as indicated by an SD within the extraocular muscle was significantly lower in $\mathrm{VMI}_{65 \mathrm{keV}}$ compared with $\mathrm{CI}$, irrespective of the $\mathrm{CTDI}_{\mathrm{vol}}(P<.001$ (Table 2 and Fig 2; eg, in 44.7-mGy protocol: $4.2 \pm 1.6$ versus $6.1 \pm 2.1$ ). Image noise slightly increased from 55 to 49.8 and $44.7 \mathrm{mGy}$, reaching a significant difference between 55- and 44.7-mGy protocols for the same reconstruction technique (eg, in CI: $5.3 \pm 1.4 \mathrm{mGy}$ versus $6.1 \pm 2.1$

Table 2: Quantitative results of attenuation, noise, and $\mathrm{CNR}^{\mathrm{a}}$

\begin{tabular}{|c|c|c|c|c|c|c|}
\hline \multirow[b]{2}{*}{$\mathrm{CTDI}_{\mathrm{vol}}(\mathrm{mGy})$} & \multicolumn{3}{|c|}{$\mathrm{Cl}$} & \multicolumn{3}{|c|}{$\mathrm{VMI}_{65 \mathrm{keV}}$} \\
\hline & 55 & 49.8 & 44.7 & 55 & 49.8 & 44.7 \\
\hline \multicolumn{7}{|l|}{ Attenuation } \\
\hline GM & $34.0 \pm 1.4$ & $33.4 \pm 1.5$ & $34.1 \pm 1.7$ & $34.6 \pm 1.3$ & $34.2 \pm 1.2$ & $35.1 \pm 1.5$ \\
\hline WM & $26.5 \pm 1.2$ & $26.1 \pm 1.5$ & $27.0 \pm 1.6$ & $26.4 \pm 1.1$ & $26.0 \pm 1.3$ & $27.1 \pm 1.4$ \\
\hline Thalamus & $33.6 \pm 1.5$ & $33.2 \pm 1.6$ & $34.4 \pm 1.9$ & $34.4 \pm 1.4$ & $33.9 \pm 1.4$ & $35.5 \pm 1.8$ \\
\hline Posterior limb & $26.5 \pm 2.2$ & $26.3 \pm 2.0$ & $26.6 \pm 2.2$ & $26.1 \pm 1.4$ & $26.0 \pm 1.7$ & $26.6 \pm 2.0$ \\
\hline Caudate nucleus & $34.5 \pm 4.4$ & $35.2 \pm 2.1$ & $35.4 \pm 2.3$ & $35.7 \pm 1.5$ & $35.8 \pm 1.8$ & $36.3 \pm 2.1$ \\
\hline Extraocular muscle & $32.8 \pm 5.6$ & $31.1 \pm 6.4$ & $33.2 \pm 8.1$ & $31.9 \pm 5.6$ & $30.8 \pm 6.2$ & $35.4 \pm 9.9$ \\
\hline Medulla oblongata & $31.5 \pm 4.3$ & $32.0 \pm 4.5$ & $34.6 \pm 4.9$ & $31.1 \pm 3.3$ & $31.0 \pm 3.0$ & $33.9 \pm 3.7$ \\
\hline \multicolumn{7}{|l|}{ Noise } \\
\hline GM & $4.5 \pm 0.8$ & $4.6 \pm 0.7$ & $4.8 \pm 0.8$ & $3.1 \pm 0.6$ & $3.1 \pm 0.5$ & $3.2 \pm 0.5$ \\
\hline WM & $4.5 \pm 0.7$ & $4.8 \pm 0.8$ & $4.9 \pm 0.7$ & $3.0 \pm 0.5$ & $3.2 \pm 0.5$ & $3.3 \pm 0.6$ \\
\hline Thalamus & $5.8 \pm 0.8$ & $6.0 \pm 1.0$ & $6.1 \pm 1.1$ & $4.0 \pm 0.7$ & $4.2 \pm 0.8$ & $4.3 \pm 0.9$ \\
\hline Posterior limb & $5.2 \pm 1.0$ & $5.5 \pm 1.1$ & $5.6 \pm 1.3$ & $3.6 \pm 0.8$ & $3.7 \pm 0.9$ & $3.9 \pm 1.0$ \\
\hline Caudate nucleus & $5.4 \pm 1.1$ & $5.6 \pm 1.2$ & $5.6 \pm 0.8$ & $3.7 \pm 0.9$ & $3.8 \pm 0.8$ & $3.8 \pm 0.6$ \\
\hline Extraocular muscle & $5.3 \pm 1.4$ & $5.6 \pm 1.6$ & $6.1 \pm 2.1$ & $3.6 \pm 1.1$ & $3.7 \pm 1.3$ & $4.2 \pm 1.6$ \\
\hline Medulla oblongata & $6.1 \pm 1.2$ & $6.4 \pm 1.2$ & $6.7 \pm 1.4$ & $4.4 \pm 1.0$ & $4.6 \pm 0.9$ & $4.7 \pm 0.9$ \\
\hline GM-WM CNR & $2.5 \pm 0.5$ & $2.4 \pm 0.5$ & $2.3 \pm 0.5$ & $3.4 \pm 0.5$ & $3.3 \pm 0.5$ & $3.2 \pm 0.5$ \\
\hline
\end{tabular}

${ }^{\text {a }}$ Results are means \pm SDs. 
mGy, $P=.004)$. Yet, image noise in $\mathrm{VMI}_{65 \mathrm{keV}}$ with the 44.7 mGy protocol was significantly lower compared with $\mathrm{CI}$ and $55 \mathrm{mGy}(4.2 \pm 1.6$ versus $5.3 \pm 1.4 \mathrm{mGy}, P<.001)$.

\section{CNR}

Overall, the CNR for gray-white matter differentiation was significantly higher in $\mathrm{VMI}_{65 \mathrm{keV}}$ compared with $\mathrm{CI}$, irrespective of $\mathrm{CTDI}_{\mathrm{vol}}(P<.001)$ (Table 2 and Fig 3). In line with noise, the CNR slightly decreased from 55 to 49.8 and $44.7 \mathrm{mGy}$, reaching a significant difference between 55- and 44.7-mGy protocols regarding the same reconstruction technique (eg, in $\mathrm{VMI}_{65 \mathrm{keV}}$ :

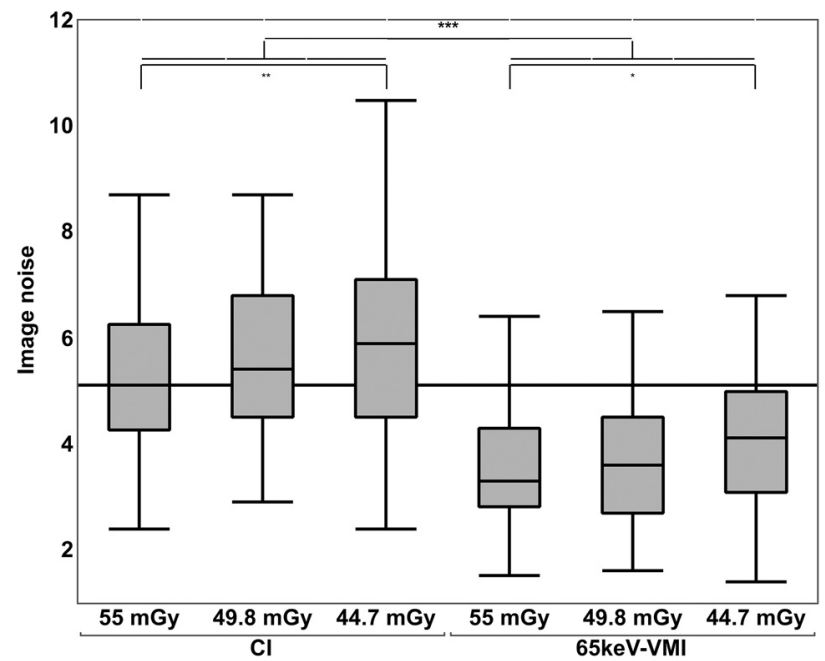

FIG 2. Image noise in extraocular muscle in $\mathrm{Cl}$ compared with 65-keV virtual monoenergetic images regarding different radiation dose protocols. Significant differences are indicated. The asterisk indicates $P=.02$; double asterisks, $P=.004$; triple asterisks, $P<.001$ ).

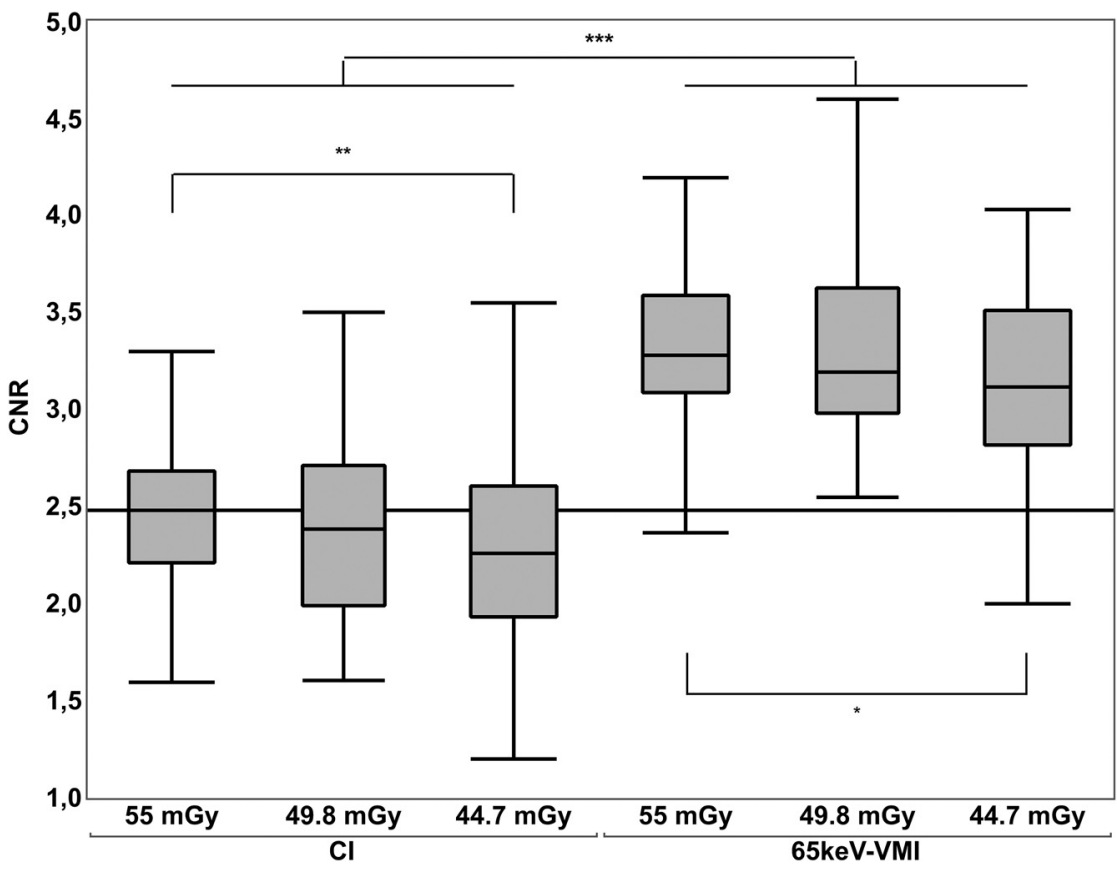

FIG 3. The CNR of gray-white matter differentiation in $\mathrm{Cl}$ compared with $65-\mathrm{keV}$ virtual monoenergetic images regarding different radiation dose protocols. Significant differences are indicated (asterisk, $P=.04$; double asterisks, $P=.02$; triple asterisks, $P<.001$ ).
$3.4 \pm 0.5$ versus $3.2 \pm 0.5 \mathrm{mGy}, P=.04$ ). Yet, the CNR in $\mathrm{VMI}_{65 \mathrm{keV}}$ with $44.7 \mathrm{mGy}$ was higher compared with CI with 55 mGy $(3.2 \pm 0.5$ versus $2.5 \pm 0.5 \mathrm{mGy}, P<.001)$.

\section{Qualitative Analysis}

The intraclass correlation between the 2 independent readers was 0.887 , indicating an excellent interreader reliability.

$\mathrm{VMI}_{65 \mathrm{kev}}$ were rated better compared with CI for all criteria (Fig 4). Irrespective of the $\mathrm{CTDI}_{\mathrm{vol}}$, gray-white matter differentiation of the basal ganglia, supra- and infratentorial corticomedullar differentiation, subjective image noise, and beam-hardening artifacts caused by the skull received superior Likert scores in $\mathrm{VMI}_{65 \mathrm{keV}}$ compared with CI $(P<.001$, Table $3)$. In the assessment of the subcalvarial space, all $\mathrm{VMI}_{65 \mathrm{keV}}$ were rated as significantly better than $\mathrm{CI}(P<.001)$, except for $\mathrm{VMI}_{65 \mathrm{keV}}$ with $49.8 \mathrm{mGy}$ compared with CI with 55 and 44.7 mGy $(P<.05)$.

\section{DISCUSSION}

This study compared the image quality of $65-\mathrm{keV}$ virtual monoenergetic images with conventional images from unenhanced spectral detector CT datasets of the head acquired with different radiation doses. We were able to show that improved image quality in $\mathrm{VMI}_{65 \mathrm{kev}}$ allows dose reduction in cranial CT.

Our study included a radiation dose reduction of $9.5 \%$ and $18.7 \%$ in terms of $\mathrm{CTDI}_{\mathrm{vol}}$. In $\mathrm{VMI}_{65 \mathrm{keV}}$, we observed significantly higher attenuation in gray matter concerning the same radiation dose and no significant differences in white matter. Image noise, on the other hand, was significantly lower compared with $\mathrm{CI}$, irrespective of the radiation dose. This reduction resulted in a significantly higher CNR for gray-white matter differentiation in $\mathrm{VMI}_{65 \mathrm{keV}}$. Hence, objective imagequality parameters were significantly better in $\mathrm{VMI}_{65 \mathrm{keV}}$ compared with $\mathrm{CI}$, irrespective of $\mathrm{CTDI}_{\mathrm{vol}}$. Accordingly, subjective image analysis indicated superiority of $\mathrm{VMI}_{65 \mathrm{keV}}$ over CI with regard to the diagnostic assessment, except for the assessment of the subcalvarial space, which was not significantly superior in all different radiation doses.

Because unenhanced cranial CT is the imaging method of choice for patients with neurologic deficits and to diagnose neurocranial traumatic lesions, there is a need for excellent image quality. ${ }^{3,30}$ At the same time, the radiation dose has to be as low as reasonably achievable because sensitive tissues are exposed. ${ }^{10,11,13}$ The observed image-quality parameters are in accordance with a recent study in which the same scanner and comparable image-acquisition parameters were used. ${ }^{6}$ Compared with a study by Pomerantz et $\mathrm{al}^{15}$ using a 


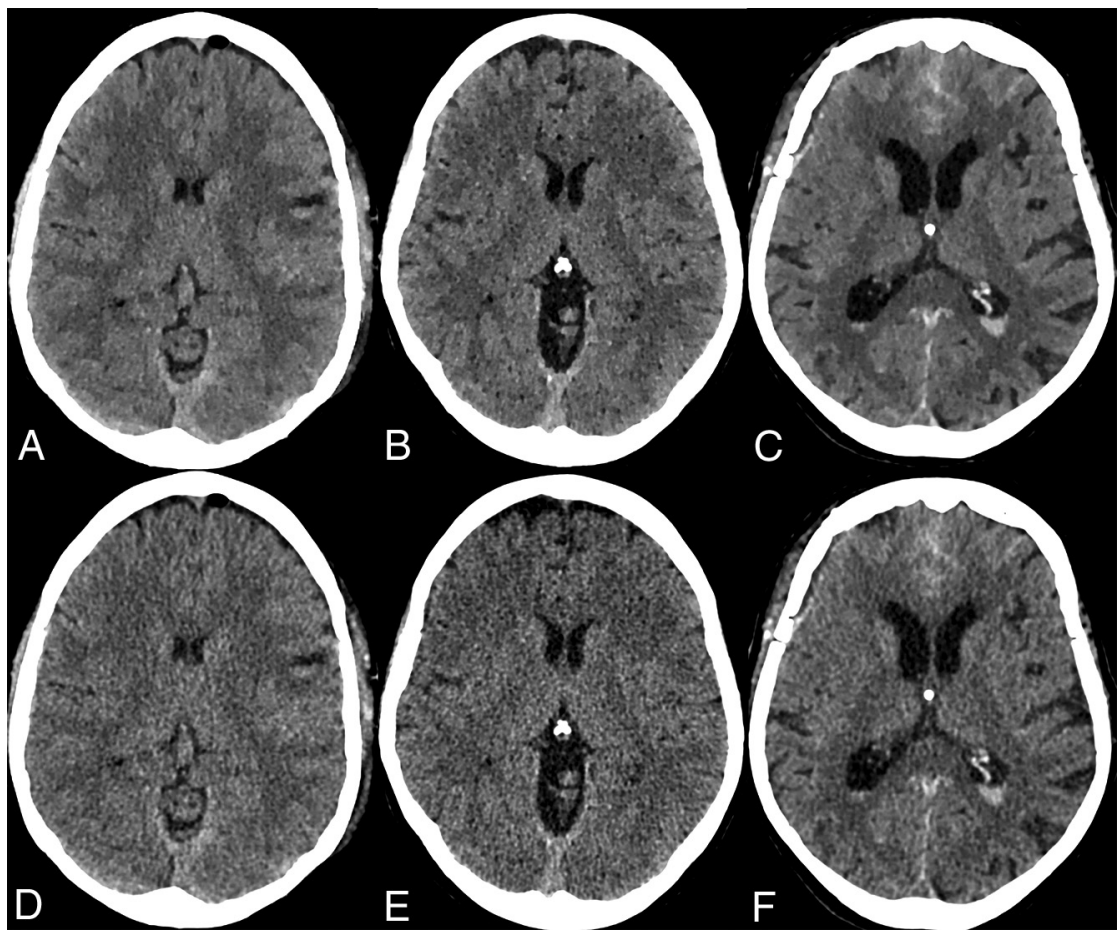

FIG 4. Examples of improved image quality in $65-\mathrm{keV}$ virtual monoenergetic images acquired with a CTDI ${ }_{\text {vol }}$ with $55 \mathrm{mGy}(A), 49.8 \mathrm{mGy}(B)$, and $44.7 \mathrm{mGy}(C)$ compared with $\mathrm{Cl}$, respectively $(D-F)$.

Table 3: Qualitative results of subjective image parameters ${ }^{a}$

\begin{tabular}{lccccccc}
\hline & \multicolumn{3}{c}{$\mathrm{Cl}$} & & \multicolumn{3}{c}{ VMI $_{\text {65keV }}$} \\
\cline { 2 - 4 } \cline { 6 - 8 } CTDI $_{\text {vol }}$ (mGy) & $\mathbf{5 5}$ & $\mathbf{4 9 . 8}$ & $\mathbf{4 4 . 7}$ & & $\mathbf{5 5}$ & $\mathbf{4 9 . 8}$ & $\mathbf{4 4 . 7}$ \\
\hline GWMA & $3(3-3)$ & $3(3-3)$ & $3(3-3)$ & & $5(5-5)$ & $5(5-5)$ & $5(4-5)$ \\
CMAS & $3(3-3)$ & $3(3-3)$ & $3(3-4)$ & & $5(5-5)$ & $5(5-5)$ & $5(5-5)$ \\
CMAI & $3(3-3)$ & $3(3-3)$ & $3(2-3)$ & & $5(5-5)$ & $5(5-5)$ & $4(4-5)$ \\
SSA & $4(4-5)$ & $4(4-4)$ & $4(4-5)$ & & $4(4-5)$ & $4(4-5)$ & $4(4-5)$ \\
Noise & $3(3-3)$ & $2(2-3)$ & $3(3-3)$ & & $4(4-4)$ & $4(4-4)$ & $4(4-4)$ \\
Artifacts & $3(3-4)$ & $3(2-3)$ & $3(2-3)$ & & $4(4-5)$ & $4(4-5)$ & $4(4-4)$ \\
\hline
\end{tabular}

Note:-GWMA indicates assessment of gray-white matter differentiation of the basal ganglia; CMAS, assessment of corticomedullary differentiation supratentorially; CMAI, assessment of corticomedullary differentiation infratentorially; SSA, assessment of subcalvarial space.

${ }^{a}$ Results are medians (quartiles).

kilovolt-switching dual-energy CT system with a $\mathrm{CTDI}_{\mathrm{vol}}$ of $72.65 \mathrm{mGy}$, our CNR values are about 1.5 times higher and image noise is slightly lower. This result is likely due to advantages regarding image noise enabled by the detector-based approach. ${ }^{24,31}$ While we compared $\mathrm{VMI}_{65 \mathrm{keV}}$ with a state-ofthe-art hybrid iterative reconstruction algorithm, whether most recent model-based image reconstructions can outperform noise reduction enabled by means of VMI remains elusive. ${ }^{32}$

So far, only a few studies have investigated dose reduction in head imaging by means of VMI compared with polychromatic CT using a kilovolt-switching dual-energy CT system. Kamiya et $\mathrm{al}^{33}$ included a radiation dose reduction of $11 \%$ in $\mathrm{VMI}_{65 \mathrm{keV}}$ compared with $\mathrm{CI}\left(\mathrm{CTDI}_{\mathrm{vol}}: 70.2 \pm 0.3 \mathrm{mGy}\right.$ versus $78.9 \pm 2.1$ mGy), while maintaining comparable image quality. However, they reported significantly higher subjective image noise in $\mathrm{VMI}_{65 \mathrm{keV}}$. On the contrary, besides an overall lower radiation dose in our study of $18.7 \%$, we yielded superior image quality quantitatively and qualitatively in $\mathrm{VMI}_{65 \mathrm{keV}}$. In line with the study by Pomerantz et al, ${ }^{15}$ Kamiya et $\mathrm{al}^{33}$ reported a lower CNR and higher noise as opposed to the results reported in this study. While the aforementioned results were reported for the supratentorial parenchyma only, we included a detailed analysis of the posterior fossa to address the most challenging region in cranial CT. Here, we report superior image quality in $\mathrm{VMI}_{65 \mathrm{keV}}$ compared with $\mathrm{CI}$, even with a reduced radiation dose.

A more recent study by Hwang et $\mathrm{al}^{34}$ investigated a radiation dose reduction of up to $37 \%$ compared with $\mathrm{CI}\left(\mathrm{CTDI}_{\mathrm{vol}}=28.0 \pm 0.9 \mathrm{mGy}\right.$ versus $44.1 \pm 1.7 \mathrm{mGy}$ ) using VMI with different kiloelectron volt values in their analyses. Yet, they conducted only a subjective analysis of image quality, reporting no significant difference. In their study, subjective overall image noise was optimal in VMI from 60 to $70 \mathrm{keV}$, which is in accordance with a few prior studies investigating optimal kiloelectron volt values for VMI in cranial CT. ${ }^{6,15}$

There are several limitations to this study. First, this was a retrospective study performed at a single institution. We were only able to include a limited number of patients because the radiation dose reduction was conducted in the run of the clinical routine; therefore, no prior power analysis was conducted. Thus, no greater reduction of the radiation dose could be evaluated, though our data suggest that this is achievable. We compared the radiation dose based on the $\mathrm{CTDI}_{\mathrm{vol}}$ alone because there is no established method to normalize radiation dose to the size of the head (unlike the size-specific dose estimates for body CT). ${ }^{35,36}$ Although our qualitative analysis was conducted in a blinded fashion, differences between $\mathrm{CI}$ and $\mathrm{VMI}_{65 \mathrm{keV}}$ are likely detectable by an experienced reader due to differences in image texture. ${ }^{32,37}$ Last, we quantitatively and qualitatively assessed image-quality parameters; however, an evaluation of diagnostic certainty and accuracy in pathologies was beyond the scope of this study.

\section{CONCLUSIONS}

The 65-keV virtual monoenergetic images from spectral detector CT enable a radiation dose reduction of $19 \%$ in cranial CT, while maintaining superior image quality over conventional images 
from full-dose acquisitions. Our data further suggest that an even greater dose reduction seems achievable.

Disclosures: Jan Borggrefe-UNRELATED: Payment for Lectures Including Service on Speakers Bureaus: Philips Healthcare, Comments: I was paid 2 times for scientific lectures in 2017 and 2018. Nils Große Hokamp_UNRELATED: Grants/Grants Pending: Philips Healthcare*; Payment for Lectures Including Service on Speakers Bureaus: Philips Healthcare; Payment for Development of Educational Presentations: Philips Healthcare. *Money paid to institution.

\section{REFERENCES}

1. Chalela JA, Kidwell CS, Nentwich LM, et al. Magnetic resonance imaging and computed tomography in emergency assessment of patients with suspected acute stroke: a prospective comparison. Lancet 2007;369:293-98 CrossRef Medline

2. Farzad A, Radin B, Oh JS, et al. Emergency diagnosis of subarachnoid hemorrhage: an evidence-based debate. J Emerg Med 2013; 44:1045-53 CrossRef Medline

3. Kim ES, Yoon DY, Lee HY, et al. Comparison of emergency cranial CT interpretation between radiology residents and neuroradiologists: transverse versus three-dimensional images. Diagn Interv Radiology 2014;20:277-84 CrossRef Medline

4. Hemphill JC, 3rd, Greenberg SM, Anderson CS, et al. Guidelines for the Management of Spontaneous Intracerebral Hemorrhage: A Guideline for Healthcare Professionals from the American Heart Association/American Stroke Association. Stroke 2015;46:2032-60 CrossRef Medline

5. Brenner DJ. Slowing the increase in the population dose resulting from CT scans. Radiat Res 2010;174:809-15 CrossRef Medline

6. Neuhaus V, Abdullayev N, Große Hokamp N, et al. Improvement of image quality in unenhanced dual-layer CT of the head using virtual monoenergetic images compared with polyenergetic singleenergy CT. Invest Radiol 2017;52:470-76 CrossRef Medline

7. Liu X, Chen L, Qi W, et al. Thin-slice brain CT with iterative model reconstruction algorithm for small lacunar lesions detection: image quality and diagnostic accuracy evaluation. Medicine (Baltimore) 2017;96:e9412 CrossRef Medline

8. Lennartz S, Laukamp KR, Neuhaus V, et al. Dual-layer detector CT of the head: Initial experience in visualization of intracranial hemorrhage and hypodense brain lesions using virtual monoenergetic images. Eur J Radiol 2018;108:177-83 CrossRef Medline

9. Neverauskiene A, Maciusovic M, Burkanas M, et al. Image based simulation of the low dose computed tomography images suggests $13 \mathrm{mAs} 120 \mathrm{kV}$ suitability for non-syndromic craniosynostosis diagnosis without iterative reconstruction algorithms. Eur J Radiol 2018;105:168-74 CrossRef Medline

10. Stewart FA, Akleyev AV, Akleyev AV, et al; Authors on Behalf of ICRP. ICRP publication 118: ICRP statement on tissue reactions and early and late effects of radiation in normal tissues and organs-threshold doses for tissue reactions in a radiation protection context. Ann ICRP 2012;41:1-322 CrossRef Medline

11. Sanchez RM, Vano E, Fernandez JM, et al. Brain radiation doses to patients in an interventional neuroradiology laboratory. AJNR Am J Neuroradiol 2014;35:1276-80 CrossRef Medline

12. Guzinski M, Waszczuk L, Sasiadek MJ. Head CT: image quality improvement of posterior fossa and radiation dose reduction with ASiR-comparative studies of CT head examinations. Eur Radiol 2016;26:3691-96 CrossRef Medline

13. Guberina N, Forsting M, Ringelstein A. Efficacy of lens protection systems: dependency on different cranial CT scans in the acute stroke setting. Radiat Prot Dosimetry 2017;175:279-83 CrossRef Medline

14. Silva AC, Morse BG, Hara AK, et al. Dual-energy (spectral) CT: applications in abdominal imaging. Radiographics 2011;31:10311046 discussion 1047-50 CrossRef Medline

15. Pomerantz SR, Kamalian S, Zhang D, et al. Virtual monochromatic reconstruction of dual-energy unenhanced head CT at $65-75 \mathrm{keV}$ maximizes image quality compared with conventional polychromatic CT. Radiology 2013;266:318-25 CrossRef Medline

16. Schneider D, Apfaltrer P, Sudarski S, et al. Optimization of kiloelectron volt settings in and cervical dual-energy CT angiography determined with virtual monoenergetic imaging. Acad Radiol 2014;21:431-36 CrossRef Medline

17. Tawfik AM, Kerl JM, Razek AA, et al. Image quality and radiation dose of dual-energy CT of the head and neck compared with a standard 120-kVp acquisition. AJNR Am J 2011;32:1994-99 CrossRef Medline

18. Bodanapally UK, Dreizin D, Issa G, et al. Dual-energy CT in enhancing subdural effusions that masquerade as subdural hematomas: diagnosis with virtual high-monochromatic (190-keV) images. AJNR Am J Neuroradiol 2017;38:1946-52 CrossRef Medline

19. Van Hedent S, Große Hokamp N, Laukamp KR, et al. Differentiation of hemorrhage from iodine using spectral detector CT: a phantom study. AJNR Am J Neuroradiol 2018;39:2205-10 CrossRef Medline

20. Kaemmerer N, Brand M, Hammon M, et al. Dual-energy computed tomography angiography of the head and neck with single-source computed tomography: a new technical (split filter) approach for bone removal. Invest Radiol 2016;51:618-23 CrossRef Medline

21. Mannil M, Ramachandran J, Vittoria de Martini I, et al. Modified dual-energy algorithm for calcified plaque removal: evaluation in carotid computed tomography angiography and comparison with digital subtraction angiography. Invest Radiol 2017;52:68085 CrossRef Medline

22. Borggrefe J, Kottlors J, Mirza M, et al. Diffrentiation of clot composition using conventional and dual-energy computed tomography. Clin Neuroadiol 2018;4:515-22 CrossRef Medline

23. Alvarez RE, Macovski A. Energy-selective reconstructions in X-ray computerized. Phys Med Biol 1976;21:733-44 CrossRef Medline

24. Große Hokamp N, Höink AJ, Doerner J, et al. Assessment of arterially hyper-enhancing liver lesions using virtual monoenergetic images from spectral detector CT: phantom and patient experience. Abdom Radiol (NY) 2018;43:2066-74 CrossRef Medline

25. Flohr TG, McCollough CH, Bruder H, et al. First performance evaluation of a dual-source CT (DSCT) system. Eur Radiol 2006;16:25668 CrossRef Medline

26. McCollough $\mathrm{CH}$, Leng $\mathrm{S}$, Yu L, et al. Dual- and multi-energy CT: principles, technical approaches, and clinical applications. Radiology 2015;276:637-53 CrossRef Medline

27. Gamer M, Lemon J, Fellows I, et al. Package irr: various coefficients of interrater reliability and agreement. 2012. https:/CRAN.Rproject.org/package.org/package-irr. Accessed March 25, 2019

28. Cohen J. A coefficient of agreement for nominal scales. Educational and Psychological Measurement 1960;20:37-46 CrossRef

29. Fleiss JL, Cohen J. The equivalence of weighted kappa and the intraclass correlation coefficient as measures of reliability. Educational and Psychological Measurement 1973;33:613-19 CrossRef

30. Smits M, Dippel DW, de Haan GG, et al. External validation of the Canadian CT Head Rule and the New Orleans Criteria for CT scanning in patients with minor head injury. JAMA 2005;294:151925 CrossRef Medline

31. Große Hokamp N, Hellerbach A, Gierich A, et al. Reduction of artifacts caused by deep brain stimulating electrodes in cranial computed tomography imaging by means of virtual monoenergetic images, metal artifact reduction algorithms, and their combination. Invest Radiol 2018;53:424-31 CrossRef Medline

32. Große Hokamp N, Gilkeson R, Jordan MK, et al. Virtual monoenergetic images from spectral detector CT as a surrogate for conventional CT images: unaltered attenuation characteristics with reduced image noise. Eur J Radiol 2019;117:49-55 CrossRef Medline

33. Kamiya $\mathrm{K}$, Kunimatsu A, Mori $\mathrm{H}$, et al. Preliminary report on virtual monochromatic spectral imaging with fast $\mathrm{kVp}$ switching dual energy head CT: comparable image quality to that of $120-\mathrm{kVp}$ CT 
without increasing the radiation dose. Jpn J Radiol 2013;31:293-98 CrossRef Medline

34. Hwang WD, Mossa-Basha M, Andre JB, et al. Qualitative comparison of noncontrast head dual-energy computed tomography using rapid voltage switching technique and conventional computed tomography. J Comput Assist Tomogr 2016;40:320-25 CrossRef Medline

35. American Association of Physicists in Medicine (TaskGroup204). Size-specific dose estimates (SSDE) in pediatric and adult body CT examinations. 2011. https://www.aapm.org/pubs/reports/detail.asp? docid=143. Accessed April 12, 2019

36. McMillan K, Bostani M, Cagnon C, et al. Size-specific, scanner-independent organ dose estimates in contiguous axial and helical head CT examinations. Med Phys 2014;41:121909 CrossRef Medline

37. Kalisz K, Rassouli N, Dhanantwari A, et al. Noise characteristics of virtual monoenergetic images from a novel detector-based spectral CT scanner. Eur J Radiol 2018;98:118-25 CrossRef Medline 